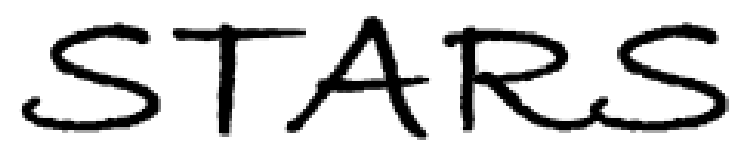

University of Central Florida

STARS

$1-1-2014$

\title{
Magnetic-field-modulated Kondo effect in a single-magnetic-ion molecule
}

Javier I. Romero

University of Central Florida

E. Vernek

E. R. Mucciolo

University of Central Florida

Find similar works at: https://stars.library.ucf.edu/facultybib2010

University of Central Florida Libraries http://library.ucf.edu

This Article is brought to you for free and open access by the Faculty Bibliography at STARS. It has been accepted for inclusion in Faculty Bibliography 2010 s by an authorized administrator of STARS. For more information, please contactSTARS@ucf.edu.

\section{Recommended Citation}

Romero, Javier I.; Vernek, E.; and Mucciolo, E. R., "Magnetic-field-modulated Kondo effect in a singlemagnetic-ion molecule" (2014). Faculty Bibliography 2010s. 6016.

https://stars.library.ucf.edu/facultybib2010/6016

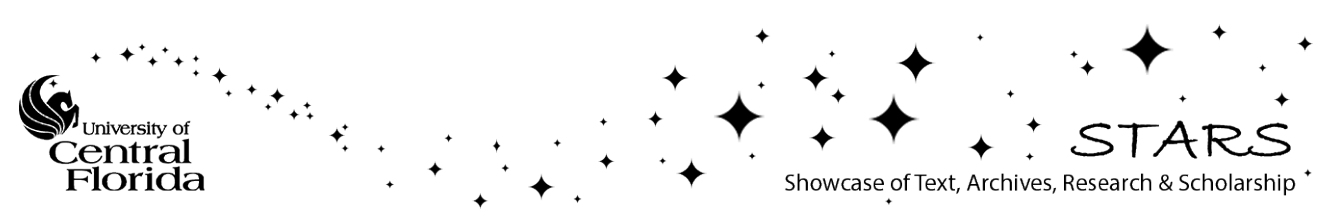




\title{
Magnetic-field-modulated Kondo effect in a single-magnetic-ion molecule
}

\author{
Javier I. Romero, ${ }^{1}$ E. Vernek, ${ }^{2,3}$ G. B. Martins, ${ }^{4}$ and E. R. Mucciolo ${ }^{1}$ \\ ${ }^{1}$ Department of Physics, University of Central Florida, Orlando, Florida 32816-2385, USA \\ ${ }^{2}$ Instituto de Física, Universidade Federal de Uberlândia, Uberlândia, Minas Gerais 38400-902, Brazil \\ ${ }^{3}$ Instituto de Física de São Carlos, Universidade de São Paulo, São Carlos, São Paulo 13560-970, Brazil \\ ${ }^{4}$ Department of Physics, Oakland University, Rochester, Michigan 48309, USA \\ (Received 14 April 2014; revised manuscript received 30 October 2014; published 13 November 2014)
}

\begin{abstract}
We study numerically the low-temperature electronic transport properties of a single-ion magnet with uniaxial and transverse spin anisotropies. We find clear signatures of a Kondo effect caused by the presence of a transverse (zero-field) anisotropy in the molecule. This Kondo effect has an SU(2) pseudospin character, associated with a doublet ground state of the isolated molecule, which results from the transverse anisotropy. Upon applying a transverse magnetic field to the single-ion magnet, we observe oscillations of the Kondo effect due to the presence of diabolical points (degeneracies) of the energy spectrum of the molecule caused by geometrical phase interference effects, similar to those observed in the quantum tunneling of multi-ion molecular nanomagnets. The field-induced lifting of the ground-state degeneracy competes with the interference modulation, resulting in some cases in a suppression of the Kondo peak.
\end{abstract}

DOI: 10.1103/PhysRevB.90.195417

PACS number(s): 75.50.Xx, 73.23.Hk, 75.45.+j, 72.10.Fk

\section{INTRODUCTION}

In recent years, there has been a growing interest in the development and study of single-ion magnets (SIMs) [1,2]. SIMs are synthesized with $3 d$ transition metal or $4 f$ rareearth single-ion magnetic centers, thus generating a simpler class of molecules whose properties are similar to those of single-molecule magnets (SMMs) [3-6]. Among their characteristics, SIMs exhibit slow spin relaxation [7,8], quantum tunneling of the magnetization [9], spin crossover regimes, and less decoherence, thus making them suitable for qubit applications [10,11]. Moreover, one of the most important characteristics of SIMs is that their magnetic anisotropies can be tailored by chemical modification of their ligands [12]. Recently, a ligand-modified SIM showed remarkable robustness in its magnetic behavior in both solid state and solution, with an observed magnetic hysteresis up to $4 \mathrm{~K}$ [13]. These properties motivate the study of electronic transport in SIMs, given the importance that nanoscale magnetic devices may have in future information technology applications.

In this work we investigate the Kondo effect in a SIM in the presence of spin anisotropies, and the effect of macroscopic spin interference effects in the tunneling of the magnetization. For these studies we employ the numerical renormalizationgroup method (NRG) [14], thus including the coupling of the SIM to the itinerant electrons in the leads in a nonperturbative and coherent way. We consider two different effective coordination geometries of the SIM: one that induces only a uniaxial spin anisotropy and another that induces both uniaxial and transverse spin anisotropies. Upon the application of a transverse magnetic field (perpendicular to the easy axis), the energy splitting of the ground and first excited states is modulated (as a function of the field magnitude) and oscillates periodically due to a Berry-phase interference effect $[15,16]$, i.e., the interference of spin tunneling paths of opposite windings.

Previous work by one of the authors studied Berry-phase oscillations of the Kondo effect in SMMs using a poor man's scaling approach [17]. In this context, the nature of the effective coupling between the itinerant electrons and the molecule magnetization was also evaluated [18]. Furthermore, nonperturbative calculations have been done to simulate the many-body charge transport properties of anisotropic magnetic impurities adsorbed onto a $\mathrm{Cu} / \mathrm{Cu}(100)$ surface [19], as well as different Kondo impurity Hamiltonians were used to model the static and dynamical properties of magnetic atoms adsorbed in nonmagnetic surfaces [20,21]. The NRG method was previously used to study the Kondo and Berryphase interference effects in the electronic transport through SMMs [22-25] (see Ref. [26] for a review). However, in those studies the interaction between the itinerant electron spin and the molecule magnetization was mainly phenomenological, namely, assumed to take the isotropic form

$$
H_{\text {Kondo }}=J_{\mathrm{K}} \vec{S}_{\mathrm{SMM}} \cdot \vec{s},
$$

where $\vec{s}$ is the itinerant electron-spin operator and $\vec{S}_{\mathrm{SMM}}$ is the total spin of the SMM. In this work we propose a microscopic model that allows us to describe the Kondo features of a SIM in terms of more fundamental parameters. For this model, we find that, at zero magnetic field, an enhancement of the conductance at low temperatures occurs whenever the SIM has a coordination geometry that induces a transverse spin anisotropy for both the ground and charged states of the molecule. The enhancement of the conductance is caused by the occurrence of a pseudospin 1/2 Kondo effect (originating from the transverse spin anisotropy), even though the total spin of the SIM is larger than $1 / 2$. Furthermore, we find that the conductance through the SIM is modulated due to interference effects in the spin tunneling paths of the SIM under an applied transverse magnetic field. As a result, the Kondo effect reemerges at a nonzero magnetic-field value that is very accessible to experiments.

The paper is organized as follows: In Sec. II we introduce the model Hamiltonian of the system. We also present the energy spectrum and eigenstates of the isolated SIM in the absence and in the presence of transverse anisotropy and magnetic field terms, highlighting the periodic modulation of the spectrum by the magnetic field. In Sec. III we evaluate 
the conductance of the molecule as a function of transverse magnetic field. In Sec. IV we summarize our conclusions.

\section{SIM MODEL AND BERRY-PHASE MODULATION OF THE ENERGY SPECTRA}

We consider a transition metal SIM consisting of an $M=1$ magnetic moment coupled to a $d$ orbital through a ferromagnetic Hund's interaction [see Fig. 1(a)]. The $d$ orbital has a spin $S_{d}=1 / 2$ when singly occupied. In addition, the total magnetic moment of the molecule, $\vec{S}=\vec{M}+\vec{S}_{d}$, has uniaxial and transverse spin anisotropies induced by a ligand environment, with the easy axis of the SIM along the $z$ direction. In the weak ligand-field regime, the anisotropy parameters arise from a perturbation of the spin-orbit coupling in the metal core using the orbital angular momentum states of the free ion (no ligands) as the unperturbed eigenstates, thus the orbital degrees of freedom are effectively integrated out in this case. The molecule is connected to two leads of noninteracting electrons only through the $d$ orbital [see Fig. 1(a)]. Here we assume that the electron transport occurs through the SIM magnetic core, thus we do not consider transport through the ligands. For simplicity, we also assume that the anisotropy does not change with the charge state of the molecule. The total Hamiltonian of the system is given by

$$
H=H_{\mathrm{M}}+H_{\text {leads }}+H_{\mathrm{ML}},
$$

where

$$
\begin{gathered}
H_{\mathrm{M}}=\sum_{\sigma} V_{g} n_{d \sigma}+\frac{U}{2} N(N-1)-J_{\mathrm{Hund}} \vec{S}_{d} \cdot \vec{M} \\
-B_{1} S_{z}^{2}+B_{2}\left(S_{x}^{2}-S_{y}^{2}\right)-h_{x} S_{x}, \\
H_{\text {leads }}=\sum_{\alpha, \mathbf{k}, \sigma} \epsilon_{\alpha \mathbf{k} \sigma} c_{\alpha \mathbf{k} \sigma}^{\dagger} c_{\alpha \mathbf{k} \sigma},
\end{gathered}
$$

and

$$
H_{\mathrm{ML}}=\sum_{\alpha, \mathbf{k}, \sigma}\left(V_{\alpha \mathbf{k}} c_{\alpha \mathbf{k} \sigma}^{+} c_{d \sigma}+\text { H.c. }\right) .
$$

In Eqs. (3)-(5), $V_{g}$ is the applied gate voltage, $U$ and $J_{\text {Hund }}$ are on-site direct and exchange Coulomb interactions, respectively, with $n_{d \sigma}$ being the number operator of the $d$ orbital and $N=\sum_{\sigma} n_{d \sigma}$. We assume that the molecule is brought to the particle-hole symmetric point through the gate voltage, therefore $V_{g}=-U / 2$. The positive coefficients $B_{1}$ and $B_{2}$ denote the easy-axis and transverse anisotropies of the components $S_{j}=M_{j}+S_{d j}(j=x, y, z)$ of the total spin $S$ of the molecule, and $h_{x}=g \mu_{B} H_{x}$ is a transverse magnetic field of amplitude $H_{x}$ applied along the $x$ axis.

Besides charge conservation in the molecule [U(1) symmetry], in the absence of the transverse anisotropy and an in-plane external magnetic field, the $z$ component of the total spin of the molecule is a good quantum number, i.e., $\left[H_{\mathrm{M}}, S_{z}\right]=0$ and $\mathrm{SU}(2)$ spin-rotational symmetry is satisfied. In this case, states labeled by the $z$ components of the total spin are also eigenstates of the Hamiltonian, and since $\left[S^{2}, S_{z}\right]=0$, the eigenstates will involve linear combinations $\left|S, S_{z}\right\rangle=$ $\sum C\left[S_{d z}, M_{z} ; S, S_{z}\right]\left|S_{d z}, M_{z}\right\rangle$, where the $C$ 's are ClebschGordan coefficients. When $B_{2}>0$, rotational symmetry is
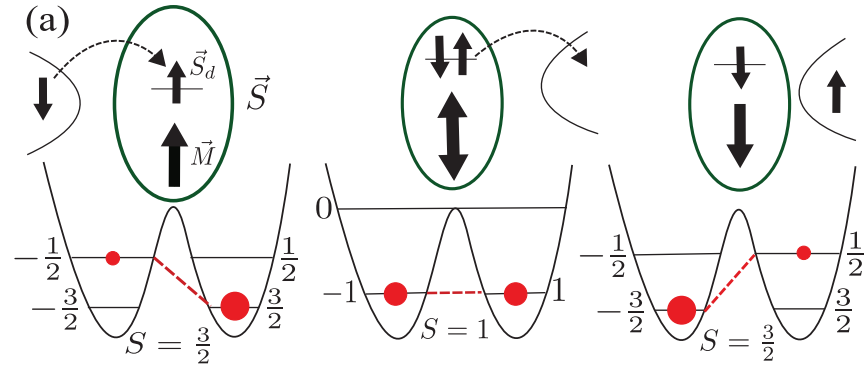

(b)

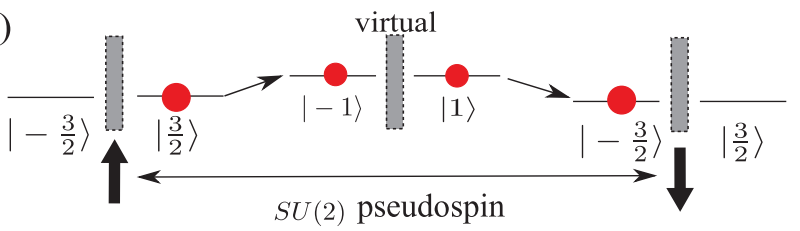

FIG. 1. (Color online) (a) Schematic representation of the SIM with cotunneling processes that result in the pseudospin SU(2) Kondo effect: spin-down electron tunnels into predominantly $|3 / 2\rangle$ SIM ground state (left), resulting in a $|-1\rangle+|1\rangle$ virtual state (center). The subsequent tunneling out of a spin-up electron takes the SIM to a predominantly $|-3 / 2\rangle$ SIM ground state (degenerate with the initial $|3 / 2\rangle$ state). A coherent sequence of these processes screens the pseudospin doublet ground state. (b) An equivalent schematic representation in the pseudospin picture, where the gray rectangle indicates the tunneling barrier between SIM states with opposite $S_{z}$ values.

broken and the eigenstates of the Hamiltonian are written as linear combinations of $\left|S, S_{z}\right\rangle$ states. Notice that even when the $\mathrm{SU}(2)$ rotational symmetry does not hold anymore, $\left[H_{\mathrm{M}}, S^{2}\right]=0$, thus $S$ is always a good quantum number.

$H_{\text {leads }}$ describes the leads, with $c_{\alpha \mathbf{k} \sigma}^{\dagger}\left(c_{\alpha \mathbf{k} \sigma}\right)$ denoting creation (annihilation) of an electron in lead $\alpha$ with energy $\epsilon_{\alpha \mathbf{k} \sigma}$, momentum $\mathbf{k}$, and spin $\sigma= \pm 1 / 2$. The tunneling term $H_{\mathrm{ML}}$ contains the hopping coupling $V_{\alpha \mathbf{k}}$ between the $d$ orbital and the leads, where $c_{d \sigma}\left(c_{\alpha \mathbf{k} \sigma}^{\dagger}\right)$ annihilates (creates) an electron with spin $\sigma$ in the SIM (lead).

Figures 2(a) and 2(b) show the magnetic-field effect on the set of eigenvalues corresponding to the charge sectors $Q=$ \pm 1 and $Q=0$, respectively (where $Q=N-1$ ). Figure 2(c) shows the tunneling splitting $\Delta=\left|E_{0}-E_{1}\right|$ between the two lowest energy states corresponding to total spin sectors $S=1$ and $S=3 / 2$. Note that these results were obtained for an isolated SIM, i.e., not connected to the leads. For a molecule described by the anisotropy terms in Eq. (3), the oscillations of the tunnel splittings in Fig. 2(c) result from a Berry-phase interference effect $[5,15]$. At zero field, spin parity dictates the nature of this interference [27]: the energy splitting is zero for a half-integer spin $(S=3 / 2)$ (the states form a Kramer's doublet) while it has a finite value for an integer $\operatorname{spin}(S=1)$, as shown in Fig. 2(c). When a magnetic field perpendicular to the easy axis is applied, time-reversal symmetry is broken and oscillations of the splitting occur. Moreover, if the direction of the magnetic field is along the hard-anisotropy axis (making an angle of 0 or $\pi$ with the $x$ axis), the interference is maximum and the splitting goes to zero at the so-called diabolical points $h_{1}$ and $h_{3 / 2}$ [28]. At these special points, seen also in the level crossings in Figs. 2(a) and 2(b), time-reversal symmetry of the corresponding spin sector is effectively restored and a change 

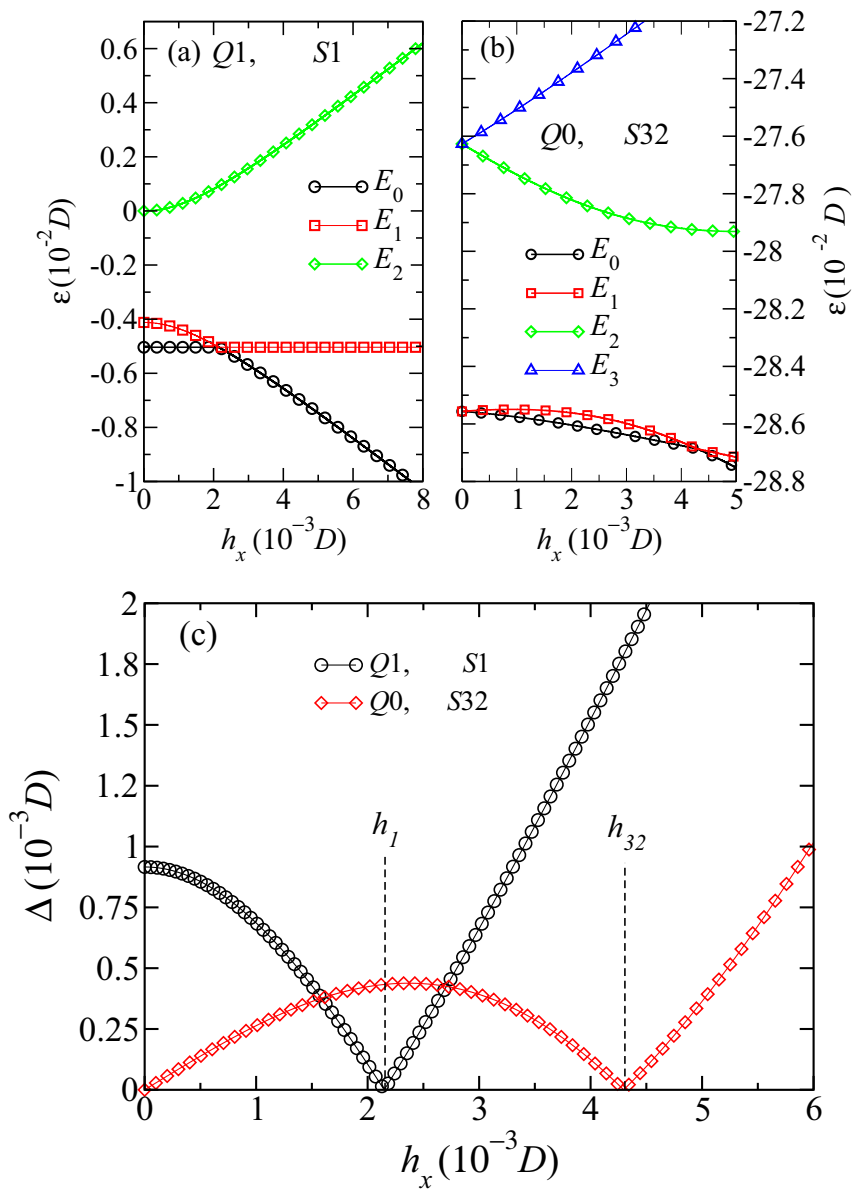

FIG. 2. (Color online) Energy eigenvalues $E_{i}$ and splitting ( $\Delta=$ $E_{1}-E_{0}$ ) for the bare molecule (not connected to the leads), where $E_{i}$ is the $i$ th eigenvalue. The interaction parameters (in units of $D$ ) are $V_{g}=-0.25, U=0.5, J_{\text {Hund }}=5 \times 10^{-2}, B_{1}=4.6 \times 10^{-3}$, and $B_{2}=4.6 \times 10^{-4}$. (a) Eigenvalues of the double occupied (or empty) $Q= \pm 1, S=1$ charge-spin sector vs $h_{x} / D$. (b) Eigenvalues of the neutral $Q=0, S=3 / 2$ charge-spin sector vs $h_{x} / D$. (c) Energy splitting $\Delta$ for the $Q= \pm 1, S=1$ [(black) circles] and $Q=0$, $S=3 / 2$ [(red) squares] charge-spin sectors.

in the symmetry of the ground and first excited spin states occurs.

\section{BERRY-PHASE MODULATION OF ELECTRONIC TRANSPORT}

We now allow the coupling of the SIM to the leads to be turned on and study the low temperature transport features of the SIM model by using the NRG method [14]. We assume that the leads have a semicircular density of states (DOS) $\rho_{\mathrm{c}}(\omega)=\left(1 / 2 \pi D^{2}\right) \sqrt{D^{2}-\omega^{2}}$, where $D$ is the half width of the conduction band, which is taken as our energy unit. Note that the use of a flat DOS leads to essentially the same results. We consider the tunneling coupling $V_{\mathbf{k}, \alpha}=V$ to be independent of $\mathbf{k}$ and equal for both leads, as well as set $k_{B}=1$. The hybridization constant $\Gamma=\pi V^{2} \rho_{\mathrm{c}}(0)$ is set to $9 \times 10^{-2} D$. In our NRG calculations, we have used a discretization parameter $\Lambda=2.5$ (keeping 3000 states at each iteration for all calculations, except for the local density
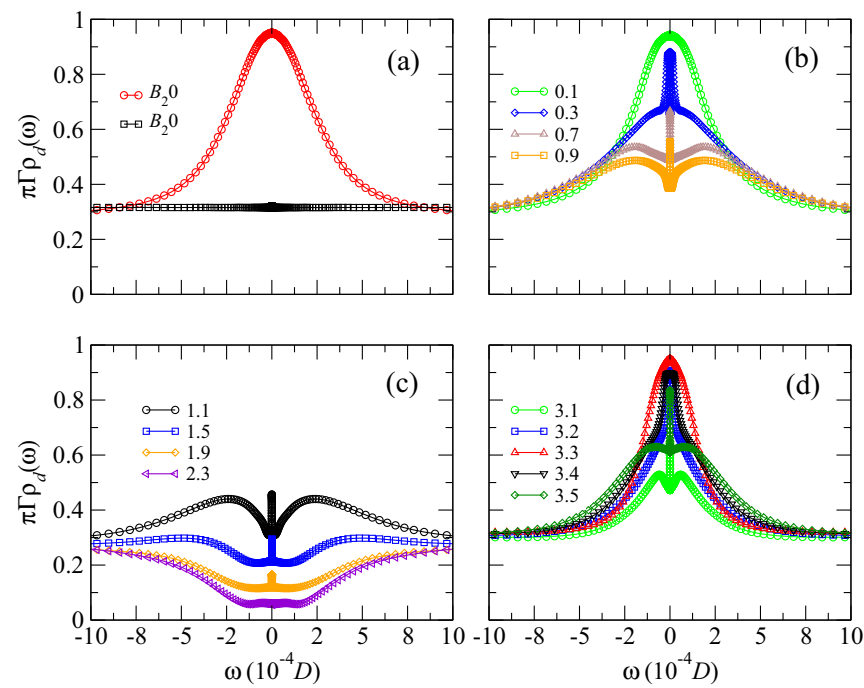

FIG. 3. (Color online) LDOS vs $\omega$ results for (a) $h_{x} / D=0$, $B_{2} / D=0$ [(black) squares] and $B_{2} / D=4.6 \times 10^{-4}$ [(red) circles]. In (b), (c), and (d) $0.1 \leqslant h_{x} /\left(10^{-3} \times D\right) \leqslant 3.5$ and $B_{2} / D=4.6 \times$ $10^{-4}$. The other interaction parameters are the same as in Fig. 2, and $\Gamma=9 \times 10^{-2} D$.

of states (LDOS) (see Fig. 3), where $\Lambda=2.0$ was used to smoothen out the oscillations around the Fermi energy. We verified that the fix point is reached before 80 sites of the Wilson's chain for both values of $\Lambda$, although we went up to 100 sites for each calculation. We also found that the phase shifts $\eta$ extracted from the numerical data were consistent with the existence of a Kondo effect $(\eta=\pi / 2)$.

The dependence of the the $d$-level LDOS with the applied transverse magnetic field around $\omega=0$ (i.e., near the Fermi energy) is shown in Fig. 3. At zero magnetic field [panel (a)], the Kondo resonance is absent for zero transverse anisotropy $\left(B_{2}=0\right)$ since there is no tunneling between the $z$ components of the virtual spin states $S_{z}= \pm 1$, thus the pseudospin flipping of the initial state cannot take place. The finite LDOS for $B_{2}=0$ in Fig. 3(a) arises because for $B_{2}=B_{1}=h_{x}=0$ the low-energy behavior of the systems corresponds to an underscreened $S=3 / 2$ Kondo model, whose LDOS presents a cusp at the Fermi level [29]. By turning on the anisotropy term $B_{1}$ this Kondo effect can be partially suppressed (for sufficiently small values of $B_{1}$ ). This has been confirmed by doubling the value of $B_{1}$, in comparison to the one used in Fig. 3(a), and noticing that the LDOS at the Fermi energy is further suppressed (not shown).

When the transverse anisotropy is nonzero, the LDOS shows the characteristic Kondo peak at the Fermi energy. In this case, one expects an enhancement of the electronic conductance through the molecule for energy scales smaller than $T_{K}$, where $T_{K}$ is the Kondo temperature. The existence of a finite transverse anisotropy $\left(B_{2}>0\right)$ in the molecule makes the virtual spin state of the system a combination of $z$ components, i.e., $|\phi\rangle=\frac{1}{\sqrt{2}}(|1\rangle-|-1\rangle)$. Thus, after an electron tunnels into the SMM, the molecule has a $50 \%$ probability of being in a spin $S_{z}= \pm 1$ state [center double well in Fig. 1(a)], making the formation of a Kondo singlet possible, through a sequence of cotunneling processes, as illustrated in Fig. 1. 

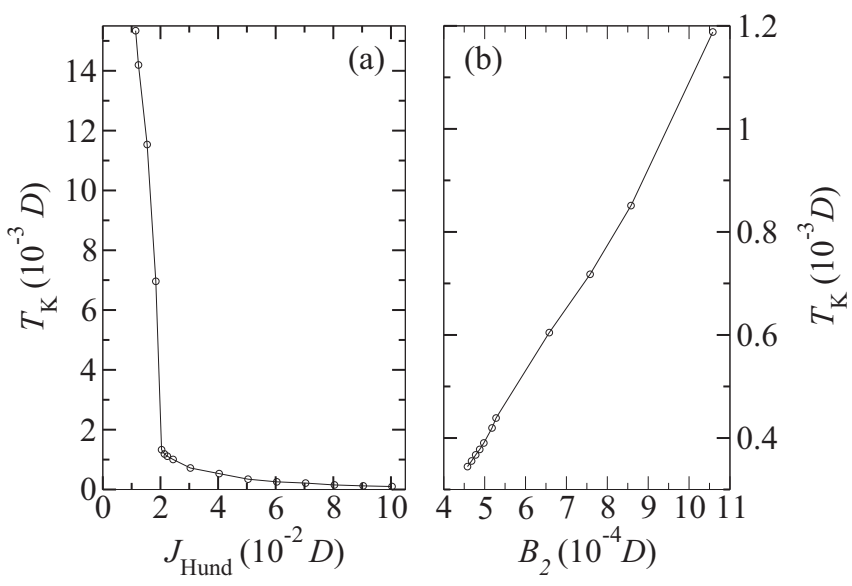

FIG. 4. Kondo temperature as a function of (a) the exchange coupling $J_{\text {Hund }}$ (for $B_{2} / D=4.6 \times 10^{-4}$ ) and (b) the transverse anisotropy parameter $B_{2}$ (for $J_{\text {Hund }} / D=5 \times 10^{-2}$ ). Other parameter values are $V_{g} / D=-0.25, U / D=0.5, B_{1} / D=4.6 \times 10^{-3}$, and $h_{x}=0 . T_{K}$ is obtained from the LDOS data as the full width at half maximum of the $\omega=0$ peak.

As the magnetic field is turned on and increased, the initial doublet ground state of the system becomes gapped in energy, and a suppression of the Kondo peak takes place, as seen in Figs. 3(b) and 3(c). Interestingly, the Kondo peak eventually resurfaces again, reaching a maximum height at about $h_{x} / D=3.3 \times 10^{-3}$ [see panel (d)]. This is because the energy gap created by the magnetic field is driven to zero at the renormalized $\tilde{h}_{3 / 2}$ diabolical point [30]. Thus, Berry-phase interference is behind the Kondo effect revival seen at this point.

When the in-plane anisotropy [the $B_{2}$ term in Eq. (4)] is strong enough, the temperature threshold for the zero-field Kondo singlet formation is $T_{K} / D \approx 5.1 \times 10^{-4}$, which corresponds to the $J_{\text {Hund }}>T_{K}$ regime. In this regime, conduction electrons see the whole spin $\vec{S}$ of the molecule [31], but only screen the doublet formed by maximum spin components of $S$, namely, a pseudospin $1 / 2$ local degree of freedom, due to the in-plane anisotropy (see Fig. 1). In the opposite case, $J_{\text {Hund }}<T_{K}$, the coupling between the local moment $\vec{M}$ and the $d$ orbital is weak and only the spin $1 / 2$ of the $d$ orbital is screened. However, this case is rather difficult to occur in practice.

The Kondo temperature is quite sensitive to the exchange coupling $J_{\text {Hund }}$ and the transverse anisotropy parameter $B_{2}$. In Fig. 4(a) we show that $T_{K}$ increases considerably as $J_{\text {Hund }}$ decreases below $2 \times 10^{-2} \mathrm{D}$. This behavior is consistent with the fact that, as $J_{\text {Hund }}$ approaches $T_{K}$, a transition in the screening regime takes place, resulting in the $d$ level being fully screened, thus causing the rapid increase of the Kondo temperature. On the other hand, as long as $B_{2}$ is large enough and the condition $J_{\text {Hund }}>T_{K}$ is fulfilled, the screening of the pseudospin $1 / 2$ dominates. Figure 4(b) shows an increase of the Kondo temperature as $B_{2}$ becomes larger. This LDOS peak widening trend with increasing transverse anisotropy is also experimentally meaningful since the molecular magnetic anisotropies can be tuned by ligand modification [12], or
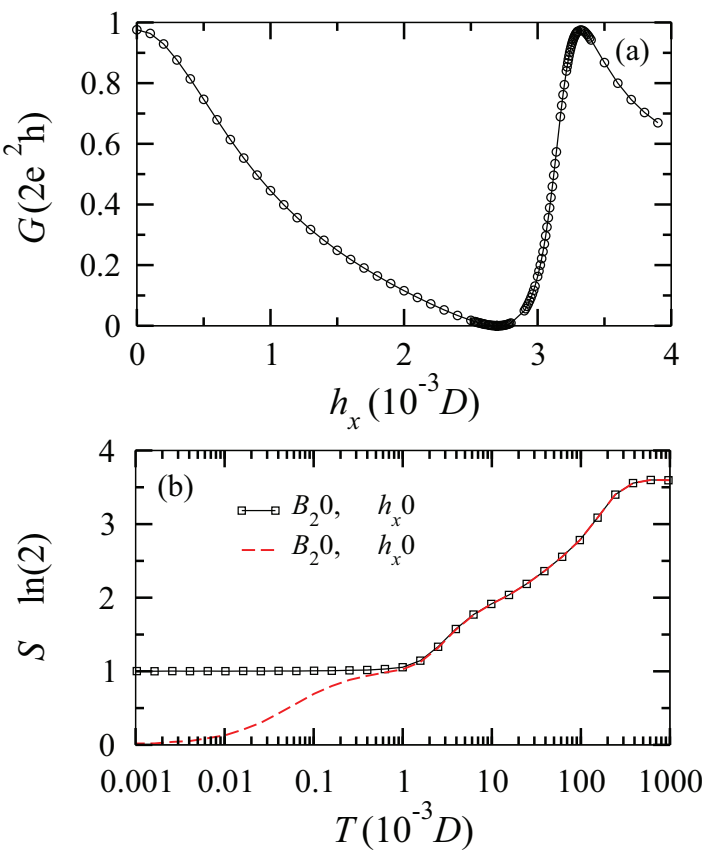

FIG. 5. (Color online) (a) Conductance through the molecule vs the transverse magnetic field $h_{x}$. (b) Entropy contribution of the SIM vs temperature.

by controlling the effective exchange coupling between the magnetic moment of the molecule and the electrodes [32].

The effect of the Berry-phase interference on the electronic transport through the SIM is seen in Fig. 5(a). The conductance is a maximum for zero field and starts to decrease as the transverse magnetic field is turned on, vanishing for $h_{x} / D \approx$ $2.7 \times 10^{-3}$. When the Berry-phase destructive interference sets in, the energy splitting of the $Q=0, S=3 / 2$ doublet goes to zero and at the $\Gamma$-renormalized diabolical point $\tilde{h}_{3 / 2} / D \approx 3.3 \times 10^{-3}$ the conductance reaches its maximum possible value again. As one moves past this point, we observe that the conductance decreases. We note that if one assumes that $D$ is likely to be of the order of a few $\mathrm{eV}$ in experimental setups, the diabolical points can be reached with relatively small fields (less than $1 \mathrm{~T}$ ).

In Fig. 5(b) we show the entropy contribution of the molecule's magnetization. At high temperatures all spin states of the molecule are available and we have $\mathcal{S}=\ln \left(2^{3.56}\right)$, which is consistent with the total number of accessible states $\Omega=12$. As the temperature decreases we can approximately keep track of the energy thresholds at which spin states of the molecule become thermally forbidden. For $T / D<6.16 \times$ $10^{-2}$, all high-energy states ( $Q= \pm 1$ sector) are suppressed. Thus, the only states available are the ones in the $Q=0$ sector. At this temperature we can have states belonging to different spin multiplets, i.e., $S=1 / 2$ or $3 / 2$, with an energy splitting induced by the exchange interaction $J_{\text {Hund }}$. Singlettriplet fluctuations are flushed away for $T / D<1.55 \times 10^{-2}$ (which is $\lesssim J_{\text {Hund }}=5 \times 10^{-2}$ ). Moreover, as we cross into the $0<T / D<8.14 \times 10^{-4}$ (which is $\lesssim B_{1}=4.6 \times 10^{-3}$ ) temperature range, the double-degenerate first excited state with spin component $S_{z}= \pm 1 / 2$ is not accessible anymore due to the uniaxial anisotropy. Then, for these low temperatures, 
the molecule can only be in the ground state $S_{z}= \pm 3 / 2$. Within this range, $B_{2}=0$ prevents the Kondo state, thus the entropy approaches $\ln 2$. For $T / D<1.5 \times 10^{-4}$, which is below the Kondo temperature, the entropy starts to approach zero since the pseudospin of the molecule is locked into a Kondo singlet with the conduction electrons. Here we also note that the temperature ranges where the Kondo effect sets in are rather accessible to experiments: for $D$ of the order of a few eV, $T_{K}$ is of the order of a few $\mathrm{K}$.

\section{CONCLUSIONS}

We have studied two interrelated effects in the electronic transport through a SIM that arise from the properties of spin anisotropies and spin-path interference behavior in this class of magnetic systems. The first effect that we have found is that an enhancement of the conductance of the molecule, namely, the Kondo effect, occurs whenever there is a ligand distortion in the SIM that, in addition to creating a strong easy-axis spin preferential direction, induces a transverse spin anisotropy in the molecule. Moreover, we observe that the lifting of the degeneracy due to the spin anisotropies transforms this problem from an underscreened total spin $S=3 / 2$ onto a fully screened pseudospin $S=1 / 2$ Kondo effect. The second effect is dependent on the first condition, and consists of the modulation of the conductance through the molecule upon the application of a transverse magnetic field. This behavior is caused by a Berry-phase interference, analogous to that observed in the quantum tunneling of the magnetization of SMM crystalline systems. Both effects are rather accessible to experiments and present an opportunity to probe the interplay between electronic transport and magnetization tunneling interference in molecular transistors.

\section{ACKNOWLEDGMENTS}

The authors acknowledge financial support from Grants No. NSF ECCS-1001755 (E.R.M.); No. NSF DMR-1107994 and No. NSF MRI-0922811 (G.B.M.); CNPq, CAPES, and FAPEMIG (E.V.). J.R. acknowledges support from the SBFAPS exchange program.
[1] N. Ishikawa, M. Sugita, T. Ishikawa, S. Koshihara, and Y. Kaizu, J. Am. Chem. Soc. 125, 8694 (2003).

[2] N. Ishikawa, Polyhedron 26, 2147 (2007).

[3] G. Christou, D. Gatteschi, D. N. Hendrickson, and R. Sessoli, MRS Bull. 25, 66 (2000).

[4] D. Gatteschi, R. Sessoli, and J. Villain, Molecular Nanomagnets (Oxford University Press, New York, 2007).

[5] W. Wernsdorfer, C. R. Chimie 11, 1086 (2008).

[6] J. R. Friedman and M. P. Sarachik, Annu. Rev. Condens. Matter Phys. 1, 109 (2010).

[7] R. Ishikawa, R. Miyamoto, H. Nojiri, B. K. Breedlove, and M. Yamashita, Inorg. Chem. 52, 8300 (2013).

[8] J. M. Zadrozny and J. R. Long, J. Am. Chem. Soc. 133, 20732 (2011).

[9] N. Ishikawa, J. Am. Chem. Soc. 127, 3650 (2005).

[10] J. Baldoví, S. Cardona-Serra, J. Clemente, E. Coronado, A. Gaita-Ariño, and A. Palii, Inorg. Chem. 51, 22 (2012).

[11] M. Martínez-Pérez et al., Phys. Rev. Lett. 108, 247213 (2012).

[12] S. Gomez-Coca, E. Cremades, N. Aliaga-Alcalde, and E. Ruiz, J. Am. Chem. Soc. 135, 7010 (2013).

[13] T. T. da Cunha et al., J. Am. Chem. Soc. 135, 16332 (2013).

[14] R. Bulla, T. A. Costi, and T. Pruschke, Rev. Mod. Phys. 80, 395 (2008).

[15] A. Garg, Europhys. Lett. 22, 205 (1993).

[16] A. Garg, E. Kochetov, K. Park, and M. Stone, J. Math. Phys. 44, 48 (2003).

[17] M. N. Leuenberger and E. R. Mucciolo, Phys. Rev. Lett. 97, 126601 (2006).
[18] G. Gonzalez, M. N. Leuenberger, and E. R. Mucciolo, Phys. Rev. B 78, 054445 (2008).

[19] R. Zitko and T. Pruschke, New J. Phys. 12, 063040 (2010).

[20] R. Zitko, R. Peters, and T. Pruschke, Phys. Rev. B 78, 224404 (2008).

[21] M. Höck and J. Schnack, Phys. Rev. B 87, 184408 (2013).

[22] C. Romeike, M. R. Wegewijs, W. Hofstetter, and H. Schoeller, Phys. Rev. Lett. 96, 196601 (2006).

[23] C. Romeike, M. R. Wegewijs, W. Hofstetter, and H. Schoeller, Phys. Rev. Lett. 97, 206601 (2006).

[24] D. Roosen, M. R. Wegewijs, and W. Hofstetter, Phys. Rev. Lett. 100, 087201 (2008).

[25] M. Misiorny, I. Weymann, and J. Barnas, Phys. Rev. B 86, 035417 (2012).

[26] M. R. Wegewijs, C. Romeike, and H. Schoeller, New J. Phys. 9, 344 (2007).

[27] D. Loss, D. P. DiVincenzo, and G. Grinstein, Phys. Rev. Lett. 69, 3232 (1992).

[28] M. V. Berry and M. Wilkinson, Proc. R. Soc. London, Ser. A 392, 15 (1984).

[29] W. Koller, A. C. Hewson, and D. Meyer, Phys. Rev. B 72, 045117 (2005).

[30] Notice that the position of the diabolical points changes once the molecule is coupled to the leads.

[31] E. Vernek, F. Qu, F. M. Souza, J. C. Egues, and E. V. Anda, Phys. Rev. B 83, 205422 (2011).

[32] J. C. Oberg, M. R. Calvo, F. Delgado, M. Moro-Lagares, D. Serrate, D. Jacob, J. Fernandez-Rossier, and C. F. Hirjibehedin, Nat. Nanotechnol. 9, 64 (2014). 